\title{
Características del uso de Internet en los cibercafés
}

\section{Characteristics of Internet use in cybercafes}

\author{
Marta Beranuy \\ Javier Fernández-Montalvo \\ Departamento de Psicología y Pedagogía, Universidad Pública de Navarra, España
}

Xavier Carbonell

Facultat de Psicologia, Ciencies de l’Educació i l’Esport Blanquerna. Universitat Ramon Llull, España

Félix Cova

Departamento de Psicología, Universidad de Concepción, Chile

Rec (02 septiembre 2015) Acept (25 enero 2016)

\begin{abstract}
Resumen
En este artículo se analizan las características del uso de Internet en una muestra de 93 jóvenes usuarios de cibercafés de la ciudad de Concepción (Chile). Fueron evaluados mediante dos instrumentos: el Cuestionario de Datos Sociodemográficos y Hábitos de Uso de Internet y el Cuestionario de Experiencias Relacionadas con Internet (CERI), que evalúa específicamente el uso problemático de la red. Los resultados mostraron que la mayor parte de los usuarios de los cibercafés eran hombres que utilizaban Internet con fines lúdicos o de comunicación. El tiempo medio de uso era de 2.5 horas por sesión, y la aplicación más utilizada era el correo electrónico. Los usuarios con puntuaciones más altas en el CERI eran aquellos que utilizaban los juegos de rol online (MMORPG) y Youtube. En estos casos, la principal motivación para el juego era de carácter social-relacional, todos eran hombres y pasaban hasta 12 horas seguidas en el cibercafé.

Palabras clave: Internet, cibercafé, abuso, adicción a Internet, Massively Multiplayer Online Role Playing Games (MMORPG).
\end{abstract}

\begin{abstract}
This article discusses the pattern of Internet use from 93 young users of cybercafés in the city of Concepción (Chile). Two instruments were administrated: a questionnaire about Sociodemographic Data and Internet habits and another one about Internet problematic use (CERI). Additionally, the main motivation for using the Internet was analyzed. The results showed that most users of cybercafés were men and the frequented this place in search of games or communication. The users spent an average of 2.5 hours per session and email was the most used service. Users with higher scores in CERI were those of role playing games (MMORPG) and youtube. The main motivation of MMORPG players was social-relational. All players were men and spent extended periods in the cybercafé (up to 12 hours).

Key words: Internet, cybercafé, abuse, Internet addiction, Massively Multiplayer Online Role Playing Games (MMORPG).
\end{abstract}




\section{Introducción}

En los últimos años, el uso y abuso de Internet, así como la existencia de la adicción a algunos servicios de éste, como las redes sociales y los juegos de rol, es un tema que ha generado muchas discusiones e, incluso, exageraciones en los medios de comunicación. En la bibliografía científica se ha estudiado desde diferentes ópticas, existiendo en los últimos años un aumento considerable del número de estudios sobre este tema (Carbonell, Guardiola, Beranuy y Bellés, 2009; Chóliz, Echeburúa y Labrador, 2012).

Los principales estudios llevados a cabo hasta la fecha indican que los jóvenes y los adolescentes constituyen un grupo de especial riesgo para abusar de la tecnología debido a la situación evolutiva en la que se encuentran (Bianchi y Phillips, 2005; Cruzado, Muñoz-Rivas y Navarro, 2001; Kamibeppu y Sugiura, 2005; Kandell, 1998; Kubey, Lavin y Barrows, 2001; Madell y Muncer, 2004; Treuer, Fábián y Füredi, 2001; Young, 1998a, 1998b). La evolución de la personalidad en esta etapa del desarrollo y la confluencia de cambios externos e internos (biológicos, psicológicos, familiares y sociales) favorece la aparición de conflictos y la focalización social, sobre todo hacia la amistad y sus rupturas (Bononato, 2005). Ello se traduce en una sensación de inseguridad. Las tecnologías de la comunicación le mantienen en sociedad, le facilitan conocer otras personas, descubrir nuevas facetas de sí mismo y, en algunos casos, facilita la expresión de sentimientos que cara a cara no se comunican (Kandell, 1998). En suma, las nuevas tecnologías le brindan un sinfín de oportunidades para probar y jugar con su identidad, aspecto fundamental en la construcción de una identidad definitiva (Matute, 2003).

Durante los últimos años se han desarrollado varios intentos de definir la adicción a Internet (Chóliz, Echeburúa y Labrador, 2012; Echeburúa, 1999; Griffiths, 2005; Greenfield, 1999; Kubey, Lavin y Barrows, 2001; Morahan-Martin y Schumacher, 2000) y se han construido diferentes instrumentos para detectarla (Anderson, 2001; Beranuy, Chamarro, Graner y Carbonell, 2009; Brenner, 1997; Caplan, 2003; de Gracia et al., 2002; Greenfield, 1999; Morahan-Martin y Schumacher, 2000; Young, 1998a y 1998b). A pesar de ello, la heterogeneidad de los instrumentos utilizados y el recurrente sesgo de selección de los estudios hace difícil estimar la prevalencia del problema (Berner y Santander, 2012). De hecho, en estos estudios el rango de la posible prevalencia de la adicción a Internet es muy amplio y oscila entre el $1.6 \%$ y el $8.2 \%$ de la población.

En la mayoría de los casos, el uso excesivo de Internet no merece probablemente el calificativo de adicción. Sin embargo, el uso abusivo de Internet comparte los elementos diagnósticos clave de otras adicciones (Griffiths y Beranuy, 2009; Sánchez-Carbonell, Beranuy, Castellana, Chamarro y Oberst, 2008): conflictos graves intra e interpersonales a consecuencia del uso de Internet, focalización, modificación del estado de ánimo, aumento de la tolerancia, síntomas de abstinencia y posibilidad de recaída. Si bien las personas pueden tener problemas en numerosos ámbitos de Internet, los Massively Multiplayer Online Role Playing Game (MMORPG) han mostrado una alta capacidad para generar adicción (Beranuy, Carbonell y Griffiths, 2013; Billieux, 2011; Cole y Griffiths, 2007; Kuss y Griffiths 2012; SánchezCarbonell et al., 2008). Como su nombre indica, se trata de juegos de rol en línea en los que participan una gran cantidad de jugadores simultáneamente. Estos juegos se organizan por objetivos y siempre hay nuevas misiones que cumplir y territorios para explorar, por lo que no tienen final. Para poder jugar es necesario crearse un personaje (denominado avatar) e interaccionar con otros. Además, muchos de los objetivos a alcanzar requieren de la cooperación de varios personajes a la vez, para lo que los jugadores deben estar conectados y jugando al mismo tiempo en grupos denominados clanes o guilds. Es necesario equipar el personaje con objetos, armas y armaduras para seguir avanzando en el contenido del juego. Esto es conocido como itemización y los jugadores pueden llegan a pagar grandes sumas de dinero para comprar personajes bien equipados. Según se avanza en los objetivos, mejora el equipamiento y, con ello, la sofisticación de las misiones, el nivel y reconocimiento del avatar, cada vez más especializado y elitista, con el consiguiente aumento de la autoestima del jugador.

Chile es uno de los países de Sudamérica más adelantado en cuanto al uso y acceso a tecnologías digitales (Universidad Católica y Centro de Estudios de Economía Digital [UC y CCS], 2009). Según los datos de Internet World Stats, web internacional que publica los datos estadísticos de la población mundial y el uso de Internet, en junio de 2012 había cerca de un $36 \%$ de usuarios de Internet en Sudamérica. En el caso concreto de Chile el 59.2\% de la población usa Internet. Constituye, por tanto, el tercer país sudamericano con más usuarios de Internet. Asimismo, varios informes sobre economía digital publicados por la cámara de comercio de Santiago de Chile (UC y CCS, 2009) muestran que han aumentado las horas semanales de conexión a Internet por parte de los usuarios. Las actividades más socorridas por los jóvenes chilenos son, por este orden, las redes sociales, el Messenger, los juegos online y el correo electrónico.

Una de las particularidades latinoamericanas en general, y de Chile en particular, es la tasa de proxy users (UC 
y CCS, 2009). Se trata de usuarios que no tienen acceso a Internet de forma directa sino a través de familiares, amigos u otros lugares. En este sentido, los cibercafés son una vía muy frecuente de acceso a Internet. Se trata de una alternativa de bajo coste, en comparación con lo que implica instalar una conexión y un ordenador en un hogar, proveen de un mejor equipo y de conexiones más rápidas y funcionan como centro de apoyo, educación y aprendizaje de nuevas herramientas virtuales (Haseloff, 2005). Además, se puede disfrutar del anonimato propio de Internet, de la fácil accesibilidad, de la huida de un mal día y de la privacidad, puesto que nadie molesta ni interfiere (Yang, Lay y Lay, 2008). Por ello, algunos autores (cfr. Martínez, Peñaranda-Cólera, Vítores e Îñiguez-Rueda, 2011) señalan que los espacios de acceso público a la tecnología como los cibercafés son enclaves esenciales para indagar el uso que se hace de Internet. Más aún si se tiene en cuenta que los cibercafés son espacios vinculados a los jóvenes y a ciertas prácticas de adicción a los juegos (Feliu, 2006). En Chile, el $21 \%$ de los internautas de 2008 se conectaba desde un espacio público (principalmente los cibercafés), siendo la mayoría de ellos de entre 12 y 29 años (UC y CCS, 2009).

A pesar de ello, los cibercafés son un espacio escasamente estudiado en Latinoamérica. Otros países lejanos, como Taiwán, por ejemplo, viven el comportamiento asociado a los cibercafés como un problema emergente para la sociedad, la escuela y las familias. Algunos estudios han mostrado que cuando los adolescentes tienen un mal día y no pueden soportar el estrés, escogen el cibercafé como vía de huida de sus problemas cotidianos, encontrando así una forma fácil y accesible de canalizar sus emociones (Yang, Lay y Lay, 2008). Los cibercafés son espacios segregados por sexo, menos visitados por las mujeres y donde los hombres tienden a jugar más (Billieux, Chanal, Khazaal, Rochat, Gay, Zullino y Van der Linden, 2011; Hsu y Chuang, 2008). Además, la población suele ser joven. En el estudio de Shah, Abdullah y Ahsan (2009), por ejemplo, más del $85 \%$ de los usuarios de cibercafés tenía menos de 30 años.

En Chile no se existe información sobre el tipo de usuarios de cibercafé, aunque sí se percibe preocupación sobre las repercusiones de Internet entre sus jóvenes (Altuzarra y Zegers, 2007; Margolis, Nussbaum, Rodríguez, Rosas, 2006; Zegers, Larraín y Trapp, 2004). Por ello, el objetivo principal de este estudio consiste en analizar el perfil de los usuarios jóvenes de los cibercafés en la ciudad universitaria de Concepción. Más en concreto, se pretende identificar las características del uso de Internet, así como las principales aplicaciones utilizadas, analizar el uso problemático de la red, conocer el uso específico que se hace de los juegos
MMORPG y determinar los motivos por los que se usa el cibercafé.

\section{Método}

\section{Participantes}

La muestra estuvo compuesta por un total de 93 sujetos (79 hombres y 14 mujeres), que fueron seleccionados con arreglo a los siguientes criterios de inclusión: a) estar en un cibercafé de la ciudad de Concepción (Chile) utilizando Internet; b) ser menor de 30 años; c) no presentar una patología grave o alteración del lenguaje que pudiera limitar la comprensión de los instrumentos de evaluación; y d) aceptar la participación en el estudio.

La edad media de la muestra fue de 19.24 años (DT = 4.09; rango: 12 - 30) y se trataba mayoritariamente de varones alumnos de secundaria. Un resumen de las principales características sociodemográficas se presenta en la tabla 1.

Tabla 1. Características sociodemográficas de la muestra $(N=93)$

\begin{tabular}{lcc}
\hline & Media & DT \\
\hline Edad media & 19,24 & 4,09 \\
\hline & & N \\
\hline Grupo de edad & & \\
$12-17$ años & 32 & $34.4 \%$ \\
$18-23$ años & 47 & $50.5 \%$ \\
$24-30$ años & 14 & $15.1 \%$ \\
\hline Sexo & & \\
Hombres & 79 & $84.9 \%$ \\
Mujeres & 14 & $15.1 \%$ \\
\hline Educación & & \\
Primaria & 12 & $12.9 \%$ \\
Secundaria & 53 & $57.0 \%$ \\
Estudios superiores en curso & 15 & $16.1 \%$ \\
Estudios superiores concluidos & 13 & $14.0 \%$ \\
\hline
\end{tabular}




\section{Instrumentos de evaluación}

Cuestionario de Experiencias Relacionadas con Internet (CERI) (Beranuy, Chamarro, Graner y Carbonell, 2009). Este cuestionario evalúa el uso problemático de Internet y, más en concreto, los conflictos intra e interpersonales relacionados con su uso. Consta de 10 ítems que se responden en una escala de tipo Likert que oscila entre 1 (nada) y 4 (mucho) en la versión original y entre 1 (nada) y 7 (mucho) en la versión utilizada en este estudio. Por tanto, el rango del cuestionario utilizado oscila entre 10 y 70. A mayor puntuación, mayor probabilidad de tener un uso problemático de Internet. El coeficiente alfa de consistencia interna para el CERI es de 0.77. En la versión original, este cuestionario se divide en dos factores. El primer factor (conflictos intrapersonales) presenta una consistencia interna de .74 e incluye 6 ítems que explican en $27.14 \%$ de la varianza; el segundo factor (conflictos interpersonales) tiene una consistencia interna de .75 e incluye 4 ítems que explican el $12.18 \%$ de la varianza.

Cuestionario de datos sociodemográficos y de hábitos de uso de Internet (instrumento no publicado). Este cuestionario de elaboración propia consta de 5 preguntas sociodemográficas y de 15 relacionadas con el uso de Internet. Estas últimas versan sobre las horas de conexión a la semana (en el cibercafé y en casa), las aplicaciones de Internet utilizadas y el orden de esas aplicaciones según el tiempo que se les dedica. Además, si juegan a MMORPG, se les pregunta por la principal motivación para ello. Dichas motivaciones están basadas en el estudio de Yee (2006): social-relacional (hablar con gente, hacer amigos), estrategia (rogar, dominar, manipular, engañar a otros), inmersión (jugar con los personajes, formar parte de sus historias), evasión (olvidar problemas, aliviar el estrés), competencia (itemizarse, optimizar ganancias, subir niveles), liderazgo (ser un líder de grupo efectivo), sumisión (tener un líder a quien seguir), aprendizaje (entender dinámicas de grupo, conocerse a uno mismo) y soledad (jugar solo, lograr los objetivos con la menor ayuda posible).

\section{Procedimiento}

Antes de comenzar la investigación, se llevó a cabo un estudio-piloto puesto que el CERI está desarrollado y validado en España y podía generar algún problema de lenguaje. Se administró a 50 personas en un cibercafé de Concepción. Tras modificar dos palabras para adecuar más el lenguaje y modificar la escala de Likert a un rango 1-7, se comprobó que los resultados se adecuaban a los estudios anteriores. Así, se procedió a la administración de los cuestionarios en la muestra que se presenta en este estudio. En concreto, los cuestionarios se administraron en siete cibercafés de la ciudad de Concepción por seis investigadores entrenados. Se establecieron distintos horarios de mañana y tarde durante seis semanas para acudir a los cibercafés más transitados. Dieciocho de los cuestionarios se administraron de noche durante dos tarreos nocturnos (vocablo típicamente chileno que se usa para denominar a los eventos que reúnen a un grupo de personas para jugar, compartir e intercambiar información, hacer amigos y aprender de otras personas sobre tecnología).

\section{Resultados}

\section{Patrón de uso de Internet}

Por lo que se refiere al patrón de uso de Internet, la media de días de conexión en el cibercafé era de 2.7 días entre semana (DT $=1.47$; rango de 0 a 5 días) y de 0.47 días durante el fin de semana ( $\mathrm{DT}=0.7$; rango de 0 a 2). Por otra parte, los sujetos de la muestra permanecían en el cibercafé una media de 2.55 horas $(\mathrm{DT}=2.6)$, con un rango amplio que oscilaba entre 0.5 y 12 horas seguidas.

En cuanto a las horas al día en las que los usuarios estaban conectados dentro y fuera del cibercafé, la media era de 4.15 horas diarias $(\mathrm{DT}=4.36)$ entre semana y 3.73 horas diarias $(\mathrm{DT}=4.88)$ durante el fin de semana.

\section{Patrón de uso de las aplicaciones}

El análisis de las principales aplicaciones que usaban los participantes puso de manifiesto que las más comunes eran, por orden de importancia, las siguientes: correo electrónico (93.5\%), Messenger (86\%), buscadores de información (80.6\%), Facebook (80.6\%) y Youtube (79.6\%). Las únicas aplicaciones que mostraron diferencias significativas en función del sexo fueron los juegos online y los juegos MMORPG, con un uso más elevado por parte de los hombres, y el tarot, que era utilizado más frecuentemente por las mujeres (tabla 2).

Por lo que se refiere a la edad, los juegos MMORPG, los juegos online y youtube fueron las aplicaciones significativamente más utilizadas por los usuarios de menor edad. Sin embargo, la banca y la bolsa fueron las aplicaciones significativamente más utilizadas por los usuarios de más edad. Las demás aplicaciones no mostraron diferencias significativas en relación con la edad de los usuarios. 
Tabla 2. Comparación del uso de las aplicaciones de Internet en función del sexo

\begin{tabular}{|c|c|c|c|c|c|c|c|}
\hline & \multicolumn{2}{|c|}{$\begin{array}{c}\text { Total } \\
(\mathrm{N}=93)\end{array}$} & \multicolumn{2}{|c|}{ Hombres $(n=79)$} & \multicolumn{2}{|c|}{ Mujeres (n=14) } & \multirow[t]{2}{*}{$\mathbf{X}^{2}$} \\
\hline & $\mathrm{N}$ & $(\%)$ & $\mathrm{N}$ & $(\%)$ & $\mathrm{N}$ & $(\%)$ & \\
\hline Correo electrónico & 87 & $(93.5)$ & 73 & $(92.4)$ & 14 & $(100)$ & 1.14 \\
\hline Messenger & 80 & $(86.0)$ & 68 & $(86.1)$ & 12 & $(85.7)$ & 0 \\
\hline Búsqueda de información & 75 & $(80.6)$ & 64 & $(81.0)$ & 11 & $(78.6)$ & 0.04 \\
\hline Facebook & 75 & $(80.6)$ & 62 & $(78.5)$ & 13 & $(92.9)$ & 1.57 \\
\hline Youtube & 74 & (79.6) & 65 & $(82.3)$ & 9 & $(64.3)$ & 2.37 \\
\hline Juegos On-line & 45 & $(48.4)$ & 44 & $(55.7)$ & 1 & $(7.1)$ & $11.23 * *$ \\
\hline Peer to peer & 45 & $(48.4)$ & 41 & (51.9) & 4 & $(28.6)$ & 2.59 \\
\hline MMORPG & 42 & $(45.2)$ & 40 & $(50.6)$ & 2 & $(14.3)$ & $6.034 *$ \\
\hline Foros & 39 & (41.9) & 34 & $(43.0)$ & 5 & $(35.7)$ & 0.26 \\
\hline Blogs-fotologs & 29 & $(31.2)$ & 24 & $(30.4)$ & 5 & $(35.7)$ & 0.16 \\
\hline Publicaciones digitales & 27 & $(29.0)$ & 23 & $(29.1)$ & 4 & $(28.6)$ & 0 \\
\hline Internet Real Chat & 26 & $(28.0)$ & 23 & $(29.1)$ & 3 & $(21.4)$ & 0.35 \\
\hline Megavideo & 25 & $(26.9)$ & 22 & $(27.8)$ & 3 & $(21.4)$ & 0.25 \\
\hline Compras & 15 & $(16.1)$ & 11 & (13.9) & 4 & $(28.6)$ & 1.89 \\
\hline Banca & 12 & $(12.9)$ & 11 & (13.9) & 1 & $(7.1)$ & 0.49 \\
\hline Tarot & 10 & $(10.8)$ & 4 & $(5.1)$ & 6 & $(42.9)$ & $17.7 * *$ \\
\hline Páginas erótico-pornográficas & 9 & $(9.7)$ & 9 & (11.4) & 0 & $(0.0)$ & 1.77 \\
\hline Bolsa & 4 & (4.3) & 3 & (3.8) & 1 & $(7.1)$ & 0.32 \\
\hline Apuestas & 1 & $(1.1)$ & 1 & (1.3) & 0 & $(0.0)$ & 0.18 \\
\hline
\end{tabular}

Nota. $* \mathrm{p}<.05 \quad * * \mathrm{p}<.001$

Tabla 3. Comparación del uso de las aplicaciones de Internet en función de la edad

\begin{tabular}{|c|c|c|c|c|c|c|c|}
\hline & \multicolumn{2}{|c|}{$\begin{array}{c}\text { 12-17 años } \\
(\mathrm{n}=32)\end{array}$} & \multicolumn{2}{|c|}{$\begin{array}{c}\text { 18-23 años } \\
(n=47)\end{array}$} & \multicolumn{2}{|c|}{$\begin{array}{c}\text { 24-30 años } \\
(n=14)\end{array}$} & \multirow[t]{2}{*}{$\mathrm{x} 2$} \\
\hline & $\mathrm{N}$ & $(\%)$ & $\mathrm{N}$ & $(\%)$ & $\mathrm{N}$ & $(\%)$ & \\
\hline Correo electrónico & 28 & $(87.5)$ & 45 & $(95.7)$ & 14 & $(100)$ & 3.28 \\
\hline Messenger & 28 & $(87.5)$ & 42 & $(89.4)$ & 10 & $(71.4)$ & 2.97 \\
\hline Búsqueda de información & 25 & $(78.1)$ & 37 & $(78.7)$ & 13 & $(92.9)$ & 1.58 \\
\hline Facebook & 23 & (71.9) & 41 & $(87.2)$ & 11 & $(78.6)$ & 2.92 \\
\hline Youtube & 29 & $(90.6)$ & 39 & $(83.0)$ & 6 & $(42.9)$ & $14.34 * * *$ \\
\hline Juegos On-line & 22 & $(68.8)$ & 20 & $(42.6)$ & 3 & $(21.4)$ & $10.03 * *$ \\
\hline Peer to peer & 19 & $(59.4)$ & 21 & $(44.7)$ & 5 & $(35.7)$ & 2.71 \\
\hline MMORPG & 20 & $(60.5)$ & 20 & $(42.6)$ & 2 & $(14.3)$ & $9.4 * *$ \\
\hline Foros & 13 & $(40.6)$ & 22 & $(46.8)$ & 4 & $(28.6)$ & 1.51 \\
\hline Blogs-fotologs & 10 & $(31.3)$ & 16 & $(34.0)$ & 3 & $(21.4)$ & 0.8 \\
\hline Publicaciones digitales & 7 & $(21.9)$ & 13 & $(27.7)$ & 7 & $(50.0)$ & 3.83 \\
\hline Internet Real Chat & 9 & $(28.1)$ & 13 & $(27.7)$ & 4 & $(28.6)$ & 0.01 \\
\hline Megavideo & 9 & $(28.1)$ & 13 & $(27.7)$ & 3 & $(21.4)$ & 0.25 \\
\hline Compras & 3 & $(9.4)$ & 7 & $(14.9)$ & 5 & $(35.7)$ & 5.1 \\
\hline Banca & 3 & $(9.4)$ & 4 & $(8.5)$ & 5 & $(35.7)$ & $7.64^{*}$ \\
\hline Tarot & 2 & $(6.3)$ & 6 & $(12.8)$ & 2 & $(14.3)$ & 1.06 \\
\hline Páginas erótico-pornográficas & 0 & $(0.0)$ & 7 & (14.9) & 2 & $(14.3)$ & 5.23 \\
\hline Bolsa & 0 & $(0.0)$ & 1 & (2.1) & 3 & (21.4) & $11.96 * *$ \\
\hline Apuestas & 0 & $(0.0)$ & 0 & $(0.0)$ & 1 & $(7.1)$ & 5.7 \\
\hline
\end{tabular}

Nota. $* \mathrm{p}<.05 \quad * * \mathrm{p}<.001$ 


\section{Motivos de uso de cibercafé}

La principal motivación para acudir a un cibercafé era no tener conexión a Internet en casa. En concreto, el 51.6\% de la muestra señalaba esta como su razón principal. Las razones por las que el $48.4 \%$ restante se encontraba en el cibercafé eran, por orden de importancia, las siguientes: 12 usuarios $(27.9 \%)$ estaban en el cibercafé por cercanía, es decir, su casa estaba lejos y tenían que hacer algún trámite o comunicarse con alguien; 11 usuarios (25.58\%) estaban en el cibercafé para jugar, es decir, preferían jugar en el cibercafé por la rápida conexión, la variedad de juegos instalados o para jugar con sus amigos; 10 usuarios (23.26\%) estaban en el cibercafé para ocupar su tiempo libre y entretenerse; 7 de ellos (16.28\%) estaban porque en casa no funcionaba el ordenador o Internet; y 3 de ellos (6.98\%) preferían el cibercafé por la intimidad que les daba.

\section{Uso problemático de Internet}

En cuanto a los problemas derivados del uso de Internet, la puntuación media en el CERI fue de 30.74 (DT = 10.59). La media del primer factor, los conflictos intrapersonales, fue de 20.28 (DT $=6.8$ ) sobre un rango posible de 6 a 42 . La media del segundo factor, los conflictos interpersonales, fue de $10.46(\mathrm{DT}=4.920)$ con un rango posible de 4 a 28. La comparación de las puntuaciones del CERI y de sus dos factores en función del sexo no mostró diferencias significativas.

Por otra parte, se observó una correlación negativa entre las puntuaciones del CERI y la edad. Estas correlaciones eran significativas para la puntuación total del CERI $(r=-0.258$; $\mathrm{p}<.05)$ y para el factor de los conflictos interpersonales $(\mathrm{r}=$ $-0.295 ; \mathrm{p}<.005)$. Sin embargo, en el factor de los conflictos intrapersonales la correlación con la edad no fue significativa $(\mathrm{r}=-0.188 ; \mathrm{p}<.070)$. Por lo tanto, cuanto más jóvenes eran los participantes, mayor probabilidad presentaban de tener problemas con el uso de Internet, principalmente en el ámbito interpersonal.

Asimismo, se comparó la puntuación del CERI entre los que usaban y no usaban cada una de las aplicaciones estudiadas. Las diferencias estadísticamente significativas se encontraron en MMORPG, youtube y correo electrónico. Como se puede observar en la tabla 4, los usuarios de MMORPG y youtube puntuaron más alto en el CERI y en sus factores. Los usuarios de correo electrónico tenían puntuaciones más bajas en todo.

\begin{tabular}{|c|c|c|c|}
\hline & Media (DT) & Media (DT) & $\mathrm{t}(\mathrm{gl})$ \\
\hline & $\begin{array}{c}\text { Usa } \\
\text { MMORPG }\end{array}$ & $\begin{array}{c}\text { No usa } \\
\text { MMORPG }\end{array}$ & \\
\hline $\begin{array}{l}\text { Puntuación } \\
\text { total del CERI }\end{array}$ & $34.34(10.8)$ & $28.25(9.8)$ & $\begin{array}{c}2.83 \\
(91)^{* *}\end{array}$ \\
\hline $\begin{array}{l}\text { Factor 1: } \\
\text { conflictos } \\
\text { intrapersonales }\end{array}$ & $22.08(6.4)$ & $19.04(6.8)$ & $2.16(91)^{*}$ \\
\hline \multirow[t]{2}{*}{$\begin{array}{c}\text { Factor 2: } \\
\text { conflictos } \\
\text { interpersonales }\end{array}$} & $12.26(5.3)$ & $9.22(4.3)$ & $\begin{array}{c}3.06 \\
(91)^{* *}\end{array}$ \\
\hline & Usa youtube & No usa youtube & \\
\hline $\begin{array}{l}\text { Puntuación } \\
\text { total del CERI }\end{array}$ & $35.40(10.7)$ & $29.03(10.1)$ & $\begin{array}{c}2.66(91) \\
* *\end{array}$ \\
\hline $\begin{array}{l}\text { Factor 1: } \\
\text { conflictos } \\
\text { intrapersonales }\end{array}$ & $23.28(6.6)$ & $19.18(6.6)$ & $\begin{array}{c}2.67 \\
(91)^{* *}\end{array}$ \\
\hline \multirow{2}{*}{$\begin{array}{c}\text { Factor 2: } \\
\text { conflictos } \\
\text { interpersonales }\end{array}$} & $12.12(5.1)$ & $9.85(4.7)$ & $2.01(91)^{*}$ \\
\hline & Usa correo-e & No usa correo-e & \\
\hline $\begin{array}{l}\text { Puntuación } \\
\text { total del CERI }\end{array}$ & $28.25(9.2)$ & 34.69 (11.5) & $\begin{array}{c}2.98 \\
(91)^{* *}\end{array}$ \\
\hline $\begin{array}{l}\text { Factor 1: } \\
\text { conflictos } \\
\text { intrapersonales }\end{array}$ & $19.04(6.5)$ & $22.25(6.9)$ & $2.27(91)^{*}$ \\
\hline $\begin{array}{l}\text { Factor 2: } \\
\text { conflictos } \\
\text { interpersonales }\end{array}$ & $9.21(4.3)$ & $12.44(5.3)$ & $\begin{array}{c}3.24 \\
(91)^{* *}\end{array}$ \\
\hline Nota. ${ }^{*} \mathrm{p}<.0$ & $* * \mathrm{p}<.01$ & & \\
\hline
\end{tabular}

\section{Uso de MMORPG}

Los 42 jugadores de MMORPG (todos hombres) jugaban, sobretodo, al WOW $(\mathrm{n}=14)$, Lineage $(\mathrm{n}=11)$ y Ragnarok $(n=6)$. Las motivaciones para jugar eran, por orden de importancia, las siguientes: social-relacional (10 usuarios, $26.3 \%$ ), competitiva ( 9 usuarios, $23.7 \%$ ), soledad (6 usuarios, $15.8 \%$ ), inmersión (5 usuarios, 13.2\%), estrategia (4 usuarios, 10.5\%), sumisión ( 3 usuarios, 7.9\%), evasión (2 usuarios, $5.3 \%$ ) y liderazgo y aprendizaje con un usuario $(2.6 \%)$ cada una.

\section{Discusión}

El principal objetivo de este estudio fue analizar el perfil de los usuarios jóvenes de los cibercafés de la ciudad chilena de Concepción. Los cibercafés constituyen un ámbito público 
donde el acceso a Internet es de gran facilidad y rapidez, y permite el anonimato de las personas conectadas. Se trata de un medio de conexión que se utiliza con gran frecuencia en Chile (UC y CCS, 2009), pero del que no se conocen con detalle las características de las personas que los frecuentan, ni el patrón específico de uso que se hace de ellos.

Los resultados obtenidos muestran que la gran mayoría de los usuarios de los cibercafés son hombres. Esta preponderancia masculina coincide con los datos proporcionados por otros estudios que se ha llevado a cabo en contextos geográficos distintos (Billieux et al., 2011; Hsu y Chuang, 2008; Shah, Abdullah y Ahsan, 2009). Además, al igual que ocurre en otros estudios (Shah, Abdullah y Ahsan, 2009), la mayoría de personas se conectaba a Internet en un cibercafé por motivos lúdicos o comunicativos.

Un ámbito en el que sí se encuentran diferencias con los resultados obtenidos en otros países se relaciona con el porcentaje de personas que tienen Internet en casa a pesar de ser usuarios de cibercafé. En Suiza, por ejemplo, la mayoría de los usuarios de cibercafé (el 87\%) dispone de Internet en casa (Billieux et al., 2011). En nuestro estudio, este porcentaje era mucho menor, ya que sólo el $48.4 \%$ de la muestra afirmaba tener conexión personal en su casa aunque acudía al cibercafé. En Chile solo el 59.2\% de la población (UC y CCS, 2009) y el 46\% de los escolares tiene ordenador en casa (Altuzarra y Zegers, 2007), cifras que indican un menor grado de penetración de Internet en los hogares en Chile a los países desarrollados. Ello determina un perfil diferente de motivaciones para el empleo de cibercafé. En Chile todavía predomina una motivación de tipo funcional dada la ausencia de acceso en el propio hogar en más de la mitad de los usuarios de cibercafés.

En cuanto al perfil de uso del cibercafé, los usuarios de este estudio permanecían conectados como media alrededor de 2.5 horas, con un rango muy amplio, que alcanza incluso las 12 horas seguidas. Esta dedicación tan intensa, que muestra un cierto grado de descontrol en algunos casos, coincide con los datos aportados en otros estudios (Joiner et al., 2012; Shah, Abdullah y Ahsan, 2009). Las principales aplicaciones utilizadas eran, por orden de importancia, correo electrónico, Messenger, buscadores de información, Facebook y Yotube. La comparación de todas estas aplicaciones en cuanto al tiempo que permanecían los usuarios de las mismas en el cibercafé, mostró que las personas que pasaban más tiempo seguido en él eran aquellos que jugaban a los juegos de rol online (MMORPG). No es ajeno a ello, probablemente, las particularidades concretas que tienen estos juegos.

Por lo que se refiere a las diferencias por sexos, se observa que los hombres se dedican más a jugar por la red y las mujeres, en cambio, utilizan más el tarot. En la bibliografía sobre el tema, es común encontrar que los hombres tiendan a utilizar Internet para jugar y las mujeres para comunicarse con otra personas o intensificar las relaciones sociales (chatear, redes sociales, etc.) (Beranuy, Oberst, Carbonell y Chamarro, 2009; Fuster, Oberst, Griffiths, Carbonell, Chamarro y Talarn, 2012; Yee, 2006). En este sentido, los resultados de nuestro estudio, junto con el de otros estudios recientes ( $c f r$. Joiner et al., 2012), respaldan la teoría de Helspar (2010), en el sentido de que las diferencias de sexo en el uso de Internet son un reflejo real de las diferencias que existen en nuestra sociedad.

El análisis de las puntuaciones obtenidas en el cuestionario CERI, como medida directa de la existencia de problemas en el uso de Internet, y su relación con las aplicaciones más utilizadas, arroja resultados interesantes. Así, se observa un uso más problemático de Internet en aquellos usuarios de MMORPG y de Youtube. Era de esperar que los juegos de rol online estuvieran relacionados con una puntuación alta en el CERI, ya que, como se ha indicado anteriormente, estos juegos tienen mucha capacidad adictiva. Sin embargo, son más sorprendentes los resultados obtenidos en este sentido con respecto a Youtube. Podría ser que los usuarios prefieran el cibercafé para descargar y ver las series televisivas porque la conexión es más rápida. Otro factor sería que Youtube tiene unas características cercanas a las de una televisión "a la carta" y generara mucha atracción entre sus usuarios puesto que facilita la evasión de la vida cotidiana. En cualquier caso, se trata de meras hipótesis explicativas, que requieren una comprobación empírica en estudios posteriores.

Por otra parte, es destacable que los usuarios de correo electrónico obtuvieron las puntuaciones más bajas en el CERI. Según Fuster et al. (2012), los juegos MMORPG son especialmente adictivos porque la comunicación que se establece en ellos permite la alteración o modificación de la identidad propia (AIC), cosa que no ocurre con el correo electrónico. En su opinión, las aplicaciones de comunicación en tiempo real donde el usuario no necesita identificarse (por ejemplo, salas de chat donde normalmente se oculta la verdadera identidad o juegos de rol en línea donde se utilizan avatares) son los que mejor explican el uso problemático y confirman las primeras especulaciones en el campo (Widyanto, Griffiths y Brunsden, 2011). En las comunicaciones que permiten la modificación de la identidad, el juego con esta puede llegar a ser más fácilmente problemático porque la vivencia de la identidad falsa tiene la capacidad de proporcionar mayor satisfacción que el verdadero yo, lo que les permite escapar de sí mismos. Desde 
esta perspectiva, Internet tiene tres usos diferenciados: la información (ya sea relacionada con el trabajo, la formación o el ocio), la comunicación (por ejemplo, redes, sociales, correo electrónico, etc.) y la alteración o modificación de identidad (por ejemplo, juegos en línea y algunos chats). Este último uso tendría un mayor riesgo de generar problemas y, en su caso más extremo, una adicción (Carbonell, Fúster, Chamarro y Oberst, 2012).

Dentro de los juegos de rol MMORPG, el juego más utilizado por los jugadores de la ciudad de Concepción fue el World of Warcraft (WOW), hecho que coincide con otros estudios al respecto (Billieux et al., 2011; Fuster et al., 2012). La aparición del WOW en 2004 marcó el comienzo de un período de escalada progresiva en el número de jugadores en línea. En la actualidad hay registrados más de 20 millones de jugadores online en todo el mundo y los jugadores de WOW representan más de la mitad de todos ellos (Woodcock, 2008). Se hacen necesarios, por tanto, más estudios que analicen las características específicas de este tipo de juegos que provocan un mayor grado de adicción entre sus usuarios.

Por último, en este estudio la principal motivación de los jugadores de MMORPG fue la social-relacional (Fuster et al., 2012; Yee, 2006). Hoy en día se sabe que las personas con riesgo de uso problemático de MMORPG son personas con tendencia a actuar precipitadamente en contextos emocionales y cuya motivación por el juego es la inmersión. En nuestro estudio un $13 \%$ de los usuarios que jugaban era por esta razón. Aun así, utilizar los MMORPG para huir de la realidad y jugar con nuevas identidades, no tiene por qué ser problemático a pesar del tiempo invertido (Wood, Griffiths y Parke, 2007; Hussian y Griffiths, 2008). Lo importante es que el juego no interfiera en las actividades importantes de la vida de la persona. De acuerdo con Griffiths (2010) un jugador puede jugar excesivamente pero no todos los jugadores que juegan en exceso pueden ser catalogados de adictos. La diferencia principal entre el exceso y la adicción tiene que ver con las consecuencias negativas del juego (Griffiths 2010; Sánchez-Carbonell, Beranuy, Castellana, Chamarro y Oberst, 2008).

Este estudio presenta algunas limitaciones que deben tenerse en cuenta en futuras investigaciones sobre el tema. En primer lugar, la muestra utilizada está compuesta por solo 93 personas. Si bien se trata de participantes de gran relevancia, principalmente debido a su validez ecológica, ya que fueron evaluados cuando se encontraban conectados a Internet en alguno de los cibercafés del estudio, sería necesario replicar los datos con muestras más amplias, en las que exista también un mayor número de representación femenina. Por otra parte, sería necesario contar también con un mayor número de personas que utilizan los juegos de rol online (los MMORPG), ya que ha mostrado ser el uso de Internet que genera una mayor problemática en nuestro estudio. De esta forma, se podrían establecer perfiles específicos de uso de las distintas aplicaciones y, especialmente, de aquellas que se relacionan con la presencia de un mayor número de problemas asociados. Al margen de todas estas limitaciones, se trata de un estudio-piloto que pretende abrir una nueva vía de investigación en el ámbito de los problemas generados por el uso de Internet en los cibercafés. Los motivos y factores que impulsan a los usuarios a acceder a Internet desde los cibercafés constituyen sin duda un campo interesante de estudio.

\section{Referencias}

Altuzarra, M. P., y Zegers, B. (2007). "Modelo empírico, descriptivo y predictivo para el compromiso de identidad en el chat en adolescentes escolares chilenos". Psykhe, 16, 85-96.

Anderson, K. (2001). "Internet use among college students: An exploratory study". Journal of American College Health, 50, 21-26.

Beranuy, M., Carbonell, X., y Griffiths, M. D. (2013). "A Qualitative Analysis of Online Gaming Addicts in Treatment". International Journal of Mental Health and Addiction, 11, 149-161.

Beranuy, M., Chamarro, A., Graner, C., y Carbonell, X. (2009). "Validación de dos escalas breves para evaluar la adicción a Internet y el abuso de móvil". Psicothema, 21, 480-485.

Beranuy, M., Oberst, U., Carbonell, X. y Chamarro, A. (2009). "Problematic Internet and mobile phone use and clinical symptoms in college students: The role of emotional intelligence". Computers in Human Behaviour, 25, 1182-1187.

Berner, J. E., y Santander J. (2012). "Abuso y dependencia de Internet: la epidemia y su controversia". Revista Chilena Neuro-Psiquiátrica, 50, 181-190.

Bianchi, A., y Phillips, J. G. (2005). "Psychological predictors of problem mobile phone use". Cyberpsychology \& Behavior, 8, 39-51.

Billieux, J., Chanal, J., Khazaal, Y., Rochat, L., Gay, P., Zullino, D., y Van der Linden, M. (2011). "Psychological predictors of problematic involvement in Massively Multiplayer Online Role-Playing Games: Illustration in a simple of male cybercafé players". Psychopathology, 44, 165-171.

Bononato, L. B. (2005). "Adicciones y nuevas tecnologías". Proyecto Hombre, 55, 17-22.

Brenner, V. (1997). "Psychology of computer use: Parameters of Internet use, abuse and addiction: the first 90 days of the Internet usage survey". Psychological Reports, 80, 879-882.

Caplan, S. E. (2003). Preference for online social interaction: A theory of problematic Internet use and psychosocial well-being. Comumunication Research, 30, 625-648.

Carbonell, X., Fúster, H., Chamarro, A., y Oberst, U. (2012). "Adicción a Internet y a móvil: Una revisión de estudios empíricos españoles". Papeles del Psicólogo, 33, 82-89.

Carbonell, X., Guardiola, E., Beranuy, M., y Belles, A. (2009). A bibliometric analysis of the scientific literature on Internet, video games, and cell phone addiction. Journal of Medical Library Association, 97, 102-107.

Chóliz, M., Echeburúa, E., y Labrador, F. J. (2012). Technological addictions: Are these the new addictions? Current Psychiatry Reviews, 8 , 290-291. 
Cole, H., y Griffiths, M. D. (2007). Social Interactions in Massively Multiplayer Online Role-Playing Gamers. Cyberpsychology \& Behavior, 10, 575583 .

Cruzado, J. A., Muñoz-Rivas, M. J., y Navarro, M. E. (2001). Adicción a Internet: de la hipotética entidad diagnóstica a la realidad clínica. Psicopatología Clínica, Legal y Forense, 1, 93-102.

de Gracia, M., Vigo, M., Fernández Pérez, M. J., y Marco, M. (2002). Problemas conductuales relacionados con el uso de Internet: Un estudio exploratorio. Anales de Psicología, 18, 273-292.

Echeburúa, E. (1999). ¿Adicciones sin drogas? Bilbao: Desclée de Brouwer.

Feliu, J. (2006). Adicción o violencia: dilemas sociales alrededor de las nuevas tecnologías y los jóvenes. En A. Gil y M. Vall-llovera (Coords.) Jóvenes en cibercafés: la dimensión fisica del futuro virtual. Barcelona: Editorial UOC.

Fuster, H., Oberst, U., Griffiths, M., Carbonell, X., Chamarro, A. y Talarn, A. (2012). Psychological motivation in online role-playing games: A study of Spanish World of Warcraft players. Anales de Psicología, 28 (1), 274-280.

Greenfield, D. N. (1999). Psychological characteristics of compulsive Internet use: a preliminary analysis. Cyberpsychology \& Behavior, 2, 403-412.

Griffiths, M. (2005). A 'components' model of addiction within a biopsychosocial framework. Journal of Substance Use, 10, 191-197.

Griffiths, M., y Beranuy, M. (2009). Adicción a los videojuegos: una breve revisión psicológica. Revista de Psicoterapia, 73, 33-49.

Griffiths, M. D. (2010). The role of context in online gaming excess and addiction: Some case study evidence. International Journal of Mental Health and Addiction, 8, 119-125.

Haseloff, A. M. (2005). Cybercafes and their potential as community development tools in India. The Journal of Community Informatics 1. Recuperado desde http://unpan1.un.org/intradoc/groups/public/ documents/APCIT\&/UNPAN023010.pdf

Helsper, E. J. (2010). Gendered Internet Use Across Generations and Life Stages. Communication Research, 37, 352-374.

Hsu, y.C., Chuang O. L. J. (2008) Are Internet Cafés Gendered Spaces? CyberPsychology \& Behavior, 11, 224-226.

Hussain, Z., y Griffiths, M.D. (2008). Gender swapping and socializing in cyberspace: An exploratory study. CyberPsychology \& Behavior, $11,47-53$.

Joiner, R., Gavin, J., Brosnan, M., Cromby, J., Gegory, H., Guiller, J., Maras, P., y Moon, A. (2012). Gender, Internet experience, Internet identification and Internet anxiety: a ten year follow-up. Cyberpsychology, Behavior, and Social Networking, 15, 370-372.

Kamibeppu, K., y Sugiura, H. (2005). Impact of the mobile phone on junior high-school students' friends hips in the Tokyo metropolitan area. Cyberpsychology \& Behavior, 8, 121-130.

Kandell, J. J. (1998). Internet addiction on campus: The vulnerability of college students. Cyberpsychology \& Behavior, 1, 11-17.

Kubey, R.W., Lavin, M. J., y Barrows, J.R. (2001) Internet use and collegiate academic performance decrements: Early findings. Journal of Communication, 51, 366-382.

Kuss, D. J., y Griffiths, M.D. (2012). Online gaming addiction in children and adolescents: A review of empirical research. Journal of Behavioral Addictions, 1, 1-20.

Madell, D., y Muncer, S. (2004). Back from the beach but hanging on the telephone? English adolescent's attitudes and experiences of mobile phones and the Internet. Cyberpsychology \& Behavior, 7, 359-367.

Margolis, J. L., Nussbaum, M., Rodriguez, P., y Rosas, R. (2006). Methodology for evaluating a novel education technology: a case study of handheld video games in Chile. Computers \& Education, 46, 174- 191.

Martínez, L. M., Peñaranda-Cólera, M. C., Vítores, A., e Íñiguez-Rueda, L. (2011). Los locutorios como espacios de integración: las tecnologías de la información y la comunicación en la construcción de redes e identidades. Psicoperspectivas, 10, 243-270.

Matute, H. (2003). Adaptarse a Internet. Galicia: La Voz de Galicia.
Morahan- Martin, J., y Schumacher, P. (2000). Incidence and correlates of pathological Internet use among college students. Computers in Human Behavior, 16, 13-29.

Sánchez-Carbonell, X., Beranuy, M., Castellana, M., Chamarro, A., y Oberst, U. (2008). La adicción a Internet y al móvil: ¿moda o trastorno? Adicciones, 20, 149-160.

Shah, S., Abdullah, Z., y Ahsan, N. (2009). Cyber Café usage in Malaysia: an exploratory study. Journal of Internet Banking \& Commerce, 14, 2-13.

Treuer, F., Fábian, Z., y Füredi, J. (2001). Internet addiction associated with features of impulse control disorder: is it a real psychiatric disorder? Journal of Affective Disorders, 66, 283.

Universidad Católica y Centro de Estudios de Economía Digital (2009). Los internautas chilenos y sus símiles en el resto del mundo. Informe de resultados WIP Chile 2008. Santiago de Chile: UC y CCS. Recuperado desde http://comunicaciones.uc.cl/prontus_fcom/site/artic/20080418/ mmedia/MULTIMEDIA 220080418230431.pdf

Widyanto, L., Griffiths, M.D. y Brunsden, V. (2011). A psychometric comparison of the Internet Addiction Test, the Internet Related Problem Scale, and Self Diagnosis. Cyberpsychology, Behavior, and Social Networking, 14 (3), 141-149.http://comunicaciones.uc.cl/prontus fcom/ site/artic/20080418/mmedia/MULTIMEDIA 220080418230431.pdf

Wood, R.T.A., Griffiths, M.D., y Parke, A. (2007). Experiences of time loss among videogame players: An empirical study. CyberPsychology \& Behavior, 10, 45-56.

Woodcock, B. (2008). An analysis of MMOG subscriptions growth, version 23.0. Recuperado desde http://www.mmogchart.com

Yang, H. J., Lay, J.S., y Lay, Y.L. (2008). Factors Affecting Cyber Café Addiction in Undergraduate Students in Taiwan. Annual Review of CyberTherapy and Telemedicine, 6, 69-75.

Yee, N. (2006). The demographics, motivations and derived experiences of users of massively-multiuser online graphical environments. PRESENCE: Teleoperators and Virtual Environments, 15, 309-329.

Young, K. (1998a). Caught in the net. New York: John Wile\& \& Sons.

Young, K. (1998b). Internet Addiction: The emergence of as new clinical disorder. Cyberpsychology \& Behavior, 1, 237-244.

Zegers, B., Larraín A. E., y Trapp, A. (2004). El Chat: ¿Medio de Comunicación o Laboratorio de Experimentación de la Identidad? Estudio en una muestra de 124 Estudiantes Universitarios de Santiago de Chile. PSYKHE, 13, 53-69. 
